\title{
Numerical Simulation of Rectangle Ice Floes Move- ment Using a Distributed Mass/Discrete Floe Model
}

\author{
by Chang Kyu Rheem, Member* \\ Hiroharu Kato, Member*
}

\author{
Hajime Yamaguchi, Member*
}

\section{Summary}

A new model, named "Distributed Mass/Discrete Floe model" is proposed for numerical simulation of short term and narrow area pack ice movement. This model possesses the advantages of both the continuum and the discrete element ones: it can express the discrete nature of pack ice which is difficult for a continuum model to treat, and it can realize much shorter computation time than a discrete element model. The pack ice is divided into ice floe bunches in which the floes, assumed to be distributed uniformly, are modeled as inelastic rectangles floating on the water. The ice interaction forces are formulated from the relation between the impulse on the bunch and the variation of the bunch momentum. The ocean flow is calculated by a multi-layer model simultaneously with the ice floe movement. The model formulation and the improvement of the computation accuracy are described in detail.

The computations were made for simplified ocean conditions driven by a uniform wind field in the marginal ice zones between the coast and off shore. The results showed some characteristics of ice and water combined flow and force acting on an ocean structure, as follows;

1. The water surface velocity distribution is distorted by the difference in the shear stress between the water/ice interface and the water/air interface. This causes the diffusion of pack ice.

3. In the case of no major current for a deep ocean, the water surface velocity can be determined from the computation only for a scale of the Ekman layer. If the water depth is less than the scale of Ekman layer, the direction of water surface flow, which is essential for the ice flow calculation, is highly affected by the water depth.

3. Many spike-like peaks appeard in the time variation of ice load on an ocean structure. This is caused by the discrete nature of the present model. Also, the results showed the rational movement of pack ice around the structure.

\section{Nomenclature}

$A_{i}$ : Total surface area of ice floes in a bunch

$C_{a}:$ Friction coefficient between the air and ice

$C_{d}:$ Friction coefficient between the air and water

$C_{w}$ : Friction cefficient between the water and ice

$C_{i}:$ Ice concentration (ratio of ice area to bunch area)

$E_{i j}:$ Eddy viscosity

$f:$ Coriolis parameter, $2 \omega \sin \phi$

$F_{x}$ : Center position of ice floe bunch in $x$-direction

$F_{y}:$ Center position of ice floe bunch in $y$-direction

$g:$ Acceleration of gravity

$h_{i}:$ Ice thickness

$\vec{k}:$ Unit vector in the depth direction

$M_{i}$ : Ice floe bunch mass

$N_{i x}$ : Number of ice floes in the bunch in $x$-direction

* University of Tokyo

Received 10th Jan. 1994

Read at the Spring meeting 18, 19th May 1994
$N_{i y}:$ Number of ice floes in the bunch in $y$-direction

$R_{x}$ : Ice floe bunch length in $x$-direction

$R_{y}$ : Ice floe bunch length in $y$-direction

$S_{x}$ : Distance between ice floes in $x$-direction

$S_{y}$ : Distance between ice floes in $y$-direction

$\overrightarrow{V_{a}}:$ Wind velocity at a height of $10 \mathrm{~m}$

$\overrightarrow{v_{i}}:$ Ice velocity

$\overrightarrow{v_{w}}:$ Water velocity

$\eta$ : Water surface elevation

$\eta^{\prime}$ : Water surface elevation due to ice displacement effect

$\mu:$ Ice/ice friction coefficient

$\rho_{a}:$ Density of air, $1.293 \mathrm{~kg} / \mathrm{m}^{3}$

$\rho_{i}:$ Density of ice, $910 \mathrm{~kg} / \mathrm{m}^{3}$

$\rho_{w}$ : Density of water, $1026 \mathrm{~kg} / \mathrm{m}^{3}$

$\overrightarrow{\tau_{b}}:$ Shear stress acting on the bottom of the layer

$\overrightarrow{\tau_{t}}$ : Shear stress acting on the top of the layer

$\phi:$ Latitude of interest

$\omega$ : Angular velocity of the earth rotation

\section{Introduction}

When movement of pack ice is simulated, it is very 
important to evaluate the interaction among the fragments of pack ice and those among the fragments and a solid body such as a coastline or an ocean structure. So far, researchers involved with such problems have treated the pack ice as a continuum. Several continuum models have been proposed and applied to long-term and wide-area predictions ${ }^{1,2), 3}$, and short-term and narrow-area predictions ${ }^{4), 5), 6\}}$. However, since the pack ice has discrete features, such models have certain limitations. On the other hand, Serror et al. ${ }^{7)}$ and Frederking \& Sayed" proposed a discrete element model approximating each ice floe by a disk. Although this approach is interesting, practical applications of it might be difficult because it requires much computation time.

A Distributed Mass/Discrete Floe model is a new model for numerical simulation of mesoscale pack ice rheology. It has the advantages of both a continuum model and a discrete element model. It can express the discrete nature of pack ice which is difficult for a continuum model to treat. It can treat larger number of floes in much shorter computation time compared to discrete element model. A rectangular floe version of this model was briefly reported in a previous paper ${ }^{9}$, and a disk floe version in other paper ${ }^{103}$. This paper describes an improved rectangular floe version. The model formulation and the improvement of the computation accuracy are described in detail.

The pack ice is divided into rectangular bunches in which the fioes, all of equal size, are assumed to be distributed uniformly. The ice interaction forces are formulated from the relation between the impulse on the ice floe bunch and the variation of the bunch momentum. The floes are modelled as inelastic rectangles floating on water. The equations of bunch motion are formulated from momentum conservation law, taking into account the Coriolis force, the sea surface inclination force, the interaction forces among the floe bunches and the stress due to the wind and water current. A semi-Lagrangian ice mass transport procedure is adopted. A multi-layer model is used in simulating the flow of sea water simultaneously with the ice floe movement to account for the surface flow more accurately. Finite difference formulations of the sea water flow have been done using the MacCormack predictor-corrector scheme.

The computations are made for several simplified ocean conditions driven by a uniform wind field in the marginal ice zones. They predict the spatial and temporal distributions of ice velocities, interaction forces, pressures and concentrations near a coast and around a structure.

\section{Ice Model}

Since the ice floes have various shapes and sizes, it is almost impossible to deal with them directly without some simplifying assumptions. In the present analysis, where the orthogonal coordinate system is adopted for the ice floes movement computation, the floes are modelled as inelastic rectangles. The pack ice is divided into rectangular ice floe bunches in which the floes, all of equal area and thickness, are assumed to be distributed uniformly. A bunch is characterized by the values of its center position, area and ice concentration which is a ratio of the ice covered area to the bunch area :

$$
C_{i}=\frac{\left(R_{x}-N_{i x} S_{x}\right)\left(R_{y}-N_{i y} S_{y}\right)}{R_{x} R_{y}}
$$

Figure 1 shows such a bunch with 4 floe elements. The inner (thick line) and outer (thin line) rectangles in the figure represent the bunch and the computational grid, respectively. The ice floes are denoted by the black rectangles in the inner rectangle. In the first rectangular floe model ${ }^{9 !}$, the most outer floes were placed along the edge of the bunch for simplicity.

\section{Equations of Bunch Motion}

Ice floes movement with a relatively short time-scale (of the order of several days at most) can be numerically well simulated with a dynamic model where the Coriolis force, the sea-surface inclination force, the interaction forces among the floe bunches and the stress due to the wind and water current are taken into account, neglecting ice growth and ablation.

The momentum change of the ice fioe bunch in a time interval of $d t$ can be expressed by the following equation :

$$
\begin{aligned}
& M_{i}\left(\vec{v}_{i}^{t+d t}-\vec{v}\right)^{\prime} \\
&= \int_{t}^{t+d t}\left(A_{i} \overrightarrow{\tau_{a}}+A_{i} \overrightarrow{\tau_{w}}-M_{i} g \nabla\left(\eta+\eta^{\prime}\right)\right. \\
&\left.-M_{i} f \vec{k} \times \overrightarrow{v_{i}}+\overrightarrow{F_{i}}\right) d t
\end{aligned}
$$

where,

$$
\begin{aligned}
& M_{i}=\rho_{i} h_{i} C_{i} R_{x} R_{y} \\
& \overrightarrow{\tau_{a}}=\rho_{a} C_{a}\left|\overrightarrow{V_{a}}-\overrightarrow{v_{i}}\right|\left(\overrightarrow{V_{a}}-\overrightarrow{v_{i}}\right) \\
& \overrightarrow{\tau_{w}}=\rho_{w} C_{w}\left|\overrightarrow{v_{w}}-\overrightarrow{v_{i}}\right|\left(\overrightarrow{v_{w}}-\overrightarrow{v_{i}}\right)
\end{aligned}
$$

$M_{i}$ is the bunch mass while $A_{i} \overrightarrow{\tau_{a}}$ and $A_{i} \overrightarrow{\tau_{w}}$ denote the shear forces acting on the bunch due to the wind and the

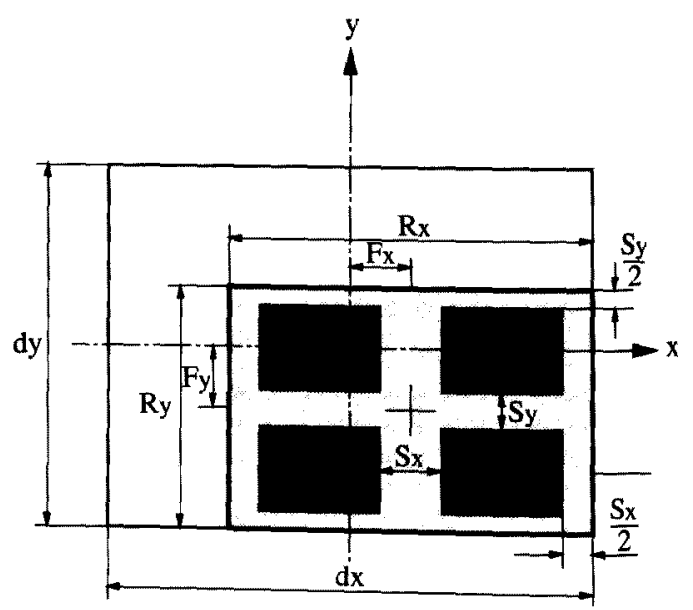

Fig. 1 Ice Floe Bunch 


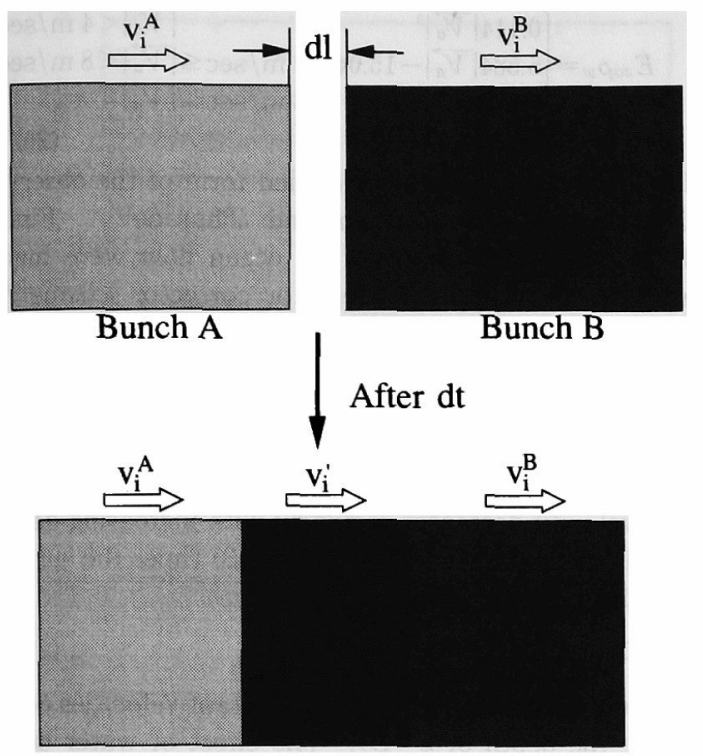

Fig. 2 Interaction between Bunches

sea current respectively. $-M_{i} g \nabla\left(\eta+\eta^{\prime}\right)$ is the force due to the inclination of sea surface. $-M_{i} f \vec{k} \times \overrightarrow{v_{i}}$ is the Coriolis force which acts in the right-hand direction perpendicular to the bunch movement in the Northern Hemisphere. $\overrightarrow{F_{i}}$ denotes the ice internal force, i. e. the interaction force of floes in a bunch and between bunches. This is the force due to the mutual collision and separation between the floes.

\subsection{Ice Internal Force}

The ice interaction forces are formulated from the relation between the impulse on the bunch and the variation of the bunch momentum, the ice floes being treated as rigid bodies. Two ice floe bunches are considered in Figure 2. The velocity of the bunch $A$ relative to bunch $B$ is expressed by,

$$
v_{s}=v_{i}^{A}-v_{i}^{B}
$$

In case that $v_{s} d t-d l-\left(S_{x}^{A}+S_{x}^{B}\right) / 2$ is positive $(d l$ is the spacing between bunches. See Fig. 1 for $S_{x}^{A}$ and $S_{x}^{B}$ ), the bunch $A$ collides with the bunch $B$ within one time step of $d t$. The number of ice floes in a bunch $A$, which take part in the collision with the neighboring bunch, is an integer $n_{x}^{A}$ satisfying the inequality,

$$
\left(n_{x}^{A}-1\right) S_{x}^{A} \leq v_{s} d t-d l-\left(S_{x}^{A}+S_{x}^{B}\right) / 2<n_{x}^{A} S_{x}^{A}
$$

$n_{x}^{A}=0$, if $n_{x}^{A}$ becomes less than 1 . Letting $n_{x}^{A} N_{i y}^{A}$ be the number of them to collide with the neighboring bunch $B$, the axial momentum change of the bunch $A$ due to the collision is expressed by,

$$
\begin{aligned}
& \int_{t}^{t+d t} F_{i x x}^{A} d t=m_{i}{ }^{A}\left(v_{i}^{\prime}-v_{i}^{A}\right) \\
& v_{i}^{\prime}=\frac{m_{i}^{A} v_{i}^{A}+m_{i}^{B} v_{i}^{B}}{m_{i}^{A}+m_{i}^{B}} \\
& m_{i}{ }^{A}=\frac{n_{x}^{A}}{N_{i x}^{A}} M_{i}^{A}
\end{aligned}
$$

where, $m_{i}^{A}$ is the mass of the floes taking part in the collision.
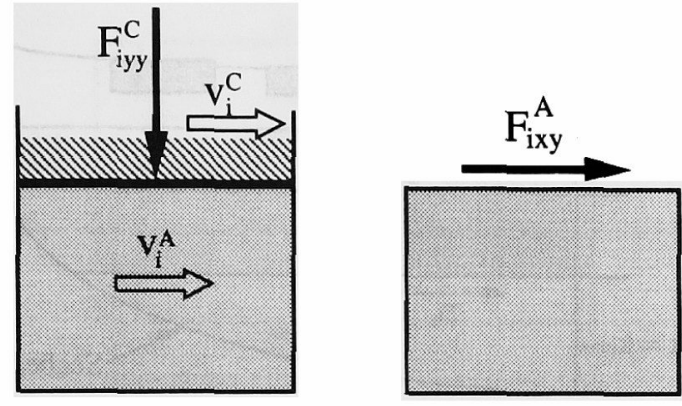

Fig. 3 Shear Force on Bunch

Let us consider the friction force on a bunch. Figure 3 shows a case where an ice floe bunch $A$ is in contact with the bunch $C . \quad F_{i y y}^{c}$ is the axial interaction force between the bunch $A$ and $C$. The shear force exerted on the ice floe bunch $A$ is expressed as,

$$
F_{i x y}^{A}=\operatorname{sign}\left(v_{i}^{C}-v_{i}^{A}\right) \mu F_{i y y}^{C}
$$

However, in case that the velocity of the bunch $A$ relative to bunch $C$ at time $t+d t$ has the opposite direction compared with that at time $t$, the direction of the shear force would be altered within one time step of $\mathrm{dt}$. In order to take into consideration the change of the direction of shear force, we construct a semi-implicit scheme.

$$
\begin{aligned}
F_{i x}^{A} d t= & \left(F_{i x x}^{A}+\operatorname{sign}\left(v_{i}^{C}-v_{i}^{A}\right) \mu F_{i y y}^{C}\right) d t^{*} \\
& +\left(F_{i x x}^{A}+\operatorname{sign}\left(v_{i}^{C}-v_{i}^{A+d t}\right) \mu F_{i y y}^{C}\right)\left(d t-d t^{*}\right)
\end{aligned}
$$

the shear force is altered. This is also an improvement from the previous version ${ }^{9}$. The effect of this improve. ment will be shown in Figure 6.

\section{2 Ice Movement and Redistribution}

The ice bunches move following the above equations. As a result, the edge of the respective bunches do not coincide with the edges of the computation mesh after the movement in the interval of $\mathrm{dt}$. Therefore, the floes are redistributed into one bunch for one mesh, conserv. ing the mass and momentum. The characteristics values of the new bunch are determined by the follow. ing equations;

$$
\begin{aligned}
& R_{x}=R_{x}^{\max }-R_{x}^{\min } \\
& R_{y}=R_{y}^{\max }-R_{y}^{\min } \\
& F_{x}=\frac{R_{x}^{\max }+R_{x}^{\min }}{2} \\
& F_{y}=\frac{R_{y}^{\max }+R_{y}^{\min }}{2} \\
& C_{i} R_{x} R_{y}=\sum_{j=1}^{n_{i}} C_{i}^{j} R_{x}^{j} R_{y}^{j} \\
& C_{i} R_{x} R_{y} \overrightarrow{v_{i}}=\sum_{j=1}^{n_{i}} C_{i}^{j} R_{x}^{j} R_{y}^{j} \overrightarrow{v_{i}^{j}}
\end{aligned}
$$

where $n_{i}$ is the number of ice bunches which came into the computation mesh under consideration (See Figure 2 in a reference 6) or Figure 3 in a reference 9)). Equations (13-5) and(13-6) express the mass and momentum conservation laws respectively. 


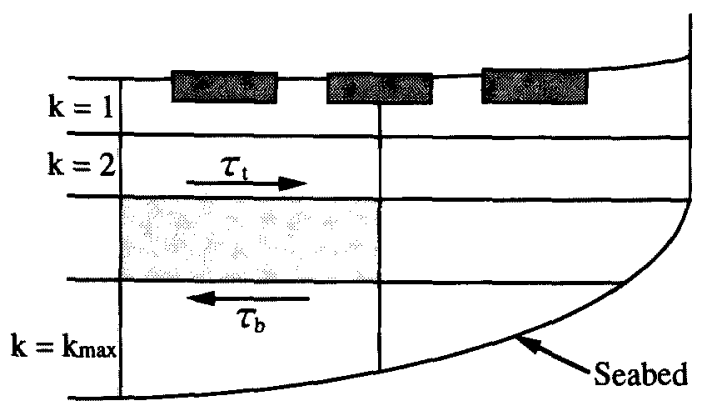

Fig. 4 Modeling of Ocean Flow

\section{3 Ocean Flow}

For the ice movement, it is important to analyze the ocean flow, particularly near the sea surface. In the present computation, the ocean flow is divided into horizontal layers as shown in Figure 4. The momentum conservation equations (the Navier-Stokes equations integrated in the layer height) and the continuity equa. tion are

$$
\begin{aligned}
& \frac{\partial q_{w x}}{\partial t}+v_{w x} \frac{\partial q_{w x}}{\partial x}+v_{w y} \frac{\partial q_{w x}}{\partial y}-f q_{w y} \\
&=-g \frac{\partial\left(\eta+\eta^{\prime}\right) d z_{k}}{\partial x}+\frac{\tau_{w x}+\tau_{b x}}{\rho_{w}}+2 E_{x x} \frac{\partial^{2} q_{w x}}{\partial x^{2}} \\
&+E_{y x} \frac{\partial}{\partial y}\left(\frac{\partial q_{w x}}{\partial x}+\frac{\partial q_{w y}}{\partial y}\right) \\
& \frac{\partial q_{w y}}{\partial t}+v_{w x} \frac{\partial q_{w y}}{\partial x}+v_{w y} \frac{\partial q_{w y}}{\partial y}+f q_{w x} \\
&=-g \frac{\partial\left(\eta+\eta^{\prime}\right) d z_{k}}{\partial y}+\frac{\tau_{t y}+\tau_{b y}}{\rho_{w}}+2 E_{y y} \frac{\partial^{2} q_{w y}}{\partial y^{2}} \\
&+E_{x y} \frac{\partial}{\partial x}\left(\frac{\partial q_{w x}}{\partial x}+\frac{\partial q_{w y}}{\partial y}\right) \\
& \frac{\partial \eta}{\partial t}+\frac{\partial Q_{w x}}{\partial x}+\frac{\partial Q_{w y}}{\partial y}=0 \\
& \text { where } d z_{k} \text { is the height of the } k \text {-th layer, and } \\
& q_{w x}=v_{w x} d z_{k}, \quad q_{w y}=v_{w y} d z_{k} \\
& Q_{w x}=\int_{-n}^{\eta} v_{w x} d z, Q_{w y}=\int_{-h}^{\eta} v_{w y} d z
\end{aligned}
$$

$q_{w x}$ and $q_{w y}$ are water fluxes in $x$-and $y$-direction in the layer, and $Q_{w x}$ and $Q_{w y}$ are total water fluxes.

For $k=1$, the shear stress on the top becomes,

$$
\overrightarrow{\tau_{t}}=C_{i} \overrightarrow{\tau_{w}}+\left(1-C_{i}\right) \overrightarrow{\tau_{a w}}
$$

where,

$$
\overrightarrow{\tau_{a w}}=\rho_{a} C_{d}\left|\overrightarrow{V_{a}}\right| \overrightarrow{V_{a}}
$$

is the shear stress between the water and air, and $\overrightarrow{\tau_{w}}$ is the stress between the water and ice given by equation (5).

The equation of $W_{u}^{11)}$ is used for the friction coefficient $C_{d}$ due to wind on the water surface.

$$
C_{d}=\left(0.8+0.065\left|\overrightarrow{V_{a}}\right|\right) \times 10^{-3}
$$

For $k=2$ to $k_{\text {wax }}$,

$$
\overrightarrow{\tau_{t}^{k+1}}=\overrightarrow{\tau_{b}^{k}}=E_{z} \frac{\partial \overrightarrow{v_{w}}}{\partial z}
$$

with the no slip condition at seabed. The eddy viscosity $E_{z}$ is obtained by multiplying grid spacing and ice concentration parameter with $E_{z o}$,

$$
E_{z o} \rho_{w}=\left\{\begin{array}{lr}
0.114\left|\overrightarrow{V_{a}}\right|^{3} & \left|\overrightarrow{V_{a}}\right|<4 \mathrm{~m} / \mathrm{sec} \\
5.584\left|\overrightarrow{V_{a}}\right|^{-15.04} & 4 \mathrm{~m} / \mathrm{sec} \leq\left|\overrightarrow{V_{a}}\right|<8 \mathrm{~m} / \mathrm{sec} \\
0.463\left|\overrightarrow{V_{a}}\right|^{2} & 8 \mathrm{~m} / \mathrm{sec} \leq\left|\overrightarrow{V_{a}}\right|
\end{array}\right\}
$$

The above equation is a modified form of the observation equations of Ekman and Thorade ${ }^{12)}$. Finite difference formulations of the ocean flow were made using the MacCormack predictor-corrector scheme ${ }^{13}$.

When the eddy viscosity is independent of depth, the sea surface wind current forms an angle of $45^{\circ}$ with the direction of the wind ${ }^{14)}$. The angle of deflection increases regularly with depth, so that at the depth $z=$ $D_{f}$ the current is directed opposite to th surface current. The velocity decreases regularly with increasing depth, and at $z=D_{f}$ it is equal to $e^{-\pi}(0.043)$ times the surface velocity.

$$
D_{f}=\pi \sqrt{\frac{2 E_{z}}{f}}
$$

This means that by far more important velocities occur above the depth of $z=D_{f}$. The effect of water depth were numerically examined by piling up the water layer. As a result, it was found that the flow below this depth of $z=D_{f}$ has little effects on the surface flow. In the present model, therefore, the water velocity is assumed to be zero below $z=D_{f}$. If a major deep water current is needed to be taken into consideration, it can be realized by giving corresponding velocity distribution as a boundary condition at $z=D_{r}$.

Figure 5 shows the results of the wind current calculation. The sea water is driven by a uniform wind field from rest, the wind velocity is $10 \mathrm{~m} / \mathrm{sec}$ and the Coriolis parameter is $0.000144 \mathrm{~s}^{-1}$, corresponding to a latitude of $80^{\circ} \mathrm{N}$. Figure 5 (a) shows the calculation results of the time variation of water surface velocities, for the case of the deep ocean (i. e., the computation water depth is $\left.D_{f}\right)$. The dotted line denotes the surface velocity obtained from the theory for a steady and infinitely deep ocean ${ }^{14)}$. The slight discrepancy between the calculation and theory is because the calculation velocities are averaged values of the top layer. The fine agreement between the two components of the calculated velocity means that the calculated surface flow forms $45^{\circ}$ with the wind direction almost exactly. Results shown in Fig. 5 ( b ) correpond to the case of the finite water depth of $20 \mathrm{~m}$. Because of the seabed effect, the deflection angle of the surface water is highly reduced, comparing with the case of deep ocean. Thus, the water depth highly affects the surface flow, which is essential for the ice motion, when the depth is less than $D_{f}$.

\section{Model Verification}

In this section, we shall apply this model to some idealized test case in order to evaluate the model's performance and demonstrate some aspects of the ice and water combined flow.

Figure $6(\mathrm{a})$ shows the first case, where uniformly distributed ice floes at the initial stage are driven by an 


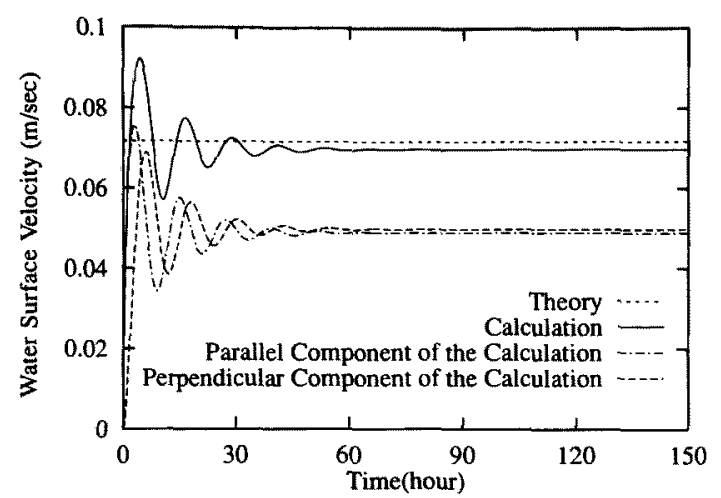

(a) Deep Ocean

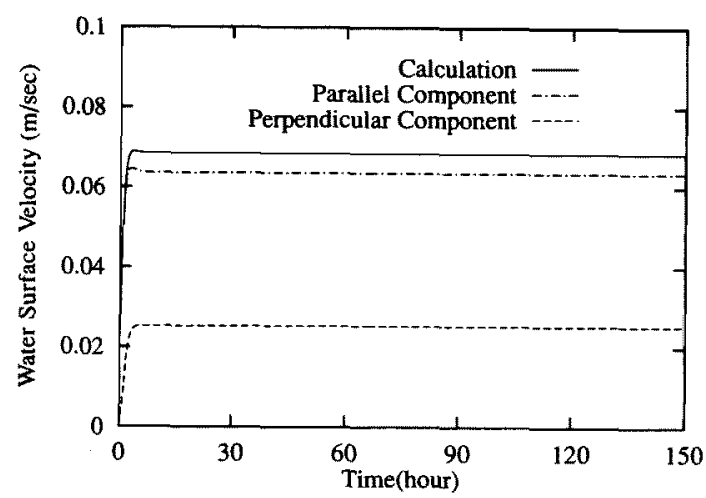

(b) Finite Depth

Fig. 5 Surface Velocity of Water Driven by a Constant Wind

uniform wind field blowing at a $45^{\circ}$ angle to the boundary. No water flow is taken into account in this case. The initial ice concentration is 0.5 and other computation parameters are given in the figure. The wind velocity is $10 \mathrm{~m} / \mathrm{sec}$, uniform in space and time. The ice floes are started from rest. The ice floes begin to move to the boundary driven by the wind, and collide with the boundary. Because they lose the momentum normal to the boundary due to the collision, they move only to one direction parallel to the boundary after the collision. The analytic solution of the steady state form of ice motion equations is easily obtained. For this condition, the analytic ice velocities parallel to the fixed boundary are $-0.1505 \mathrm{~m} / \mathrm{sec}$ and $-0.1213 \mathrm{~m} / \mathrm{sec}$. The former is that after the collision, and the latter before collision. Figure $6(\mathrm{~b})$ illustrates the variation of ice velocity distribution along the distance from the fixed boundary after 3 hours when the semi-implicit sheme (equatin (12)) is used for time integral. It shows that the ice velocity is independent of the time interval of numerical integration. The velocities after the collision (distance < about $1400 \mathrm{~m}$ ) and before the collision (distance $>$ about $1600 \mathrm{~m})$ agree very well with the abovementioned analytical values. Figure $6(\mathrm{c})$ shows the

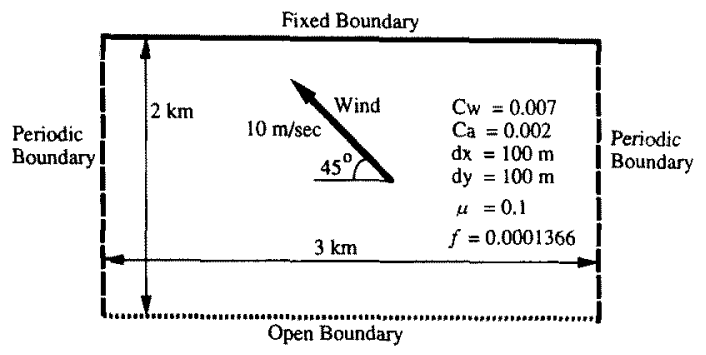

(a) Computation Domain and Boundary Conditions

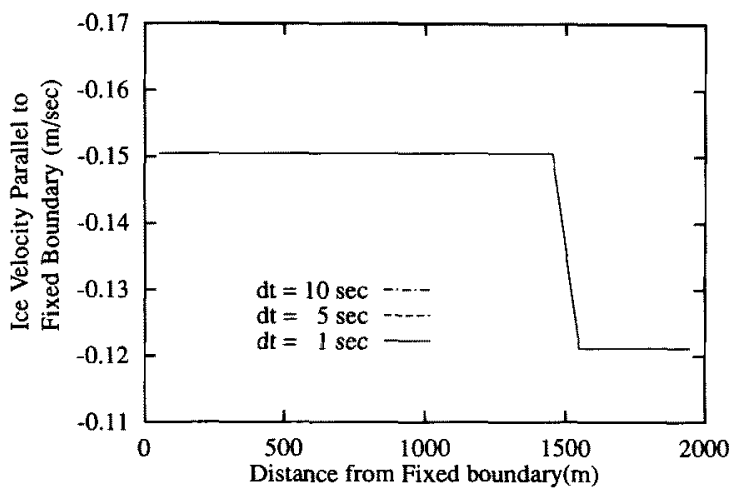

(b) Semi-Implicit Scheme

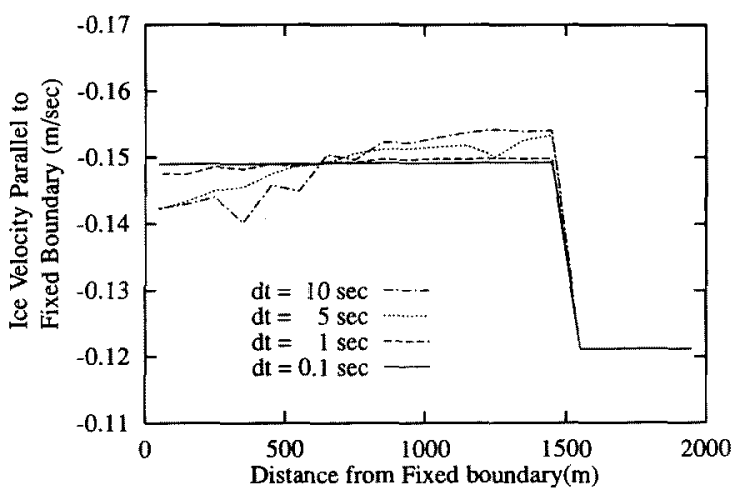

(c) Fully Explicit Scheme

Fig. 6 Effect of Time Integral Method; Comparison of Distribution of Ice Velocity Parallel to the Fixed Boundary (Positive in the Upwind Direction) After 3 Hours; No Water Flow Taken into Account

result of the fully explicit scheme (equation (11)), where the result depends on the time interval of integra. tion and only the result of $d t=0.1 \mathrm{sec}$ agrees with those of Figure $6(\mathrm{~b})$. These results show that the use of the semi-implicit scheme increases considerably the allow. able time step compared with a fully explicit scheme.

The second test case is the simulation of wind driven ice movement and spread in a simplified $50 \mathrm{~m}$ deep 


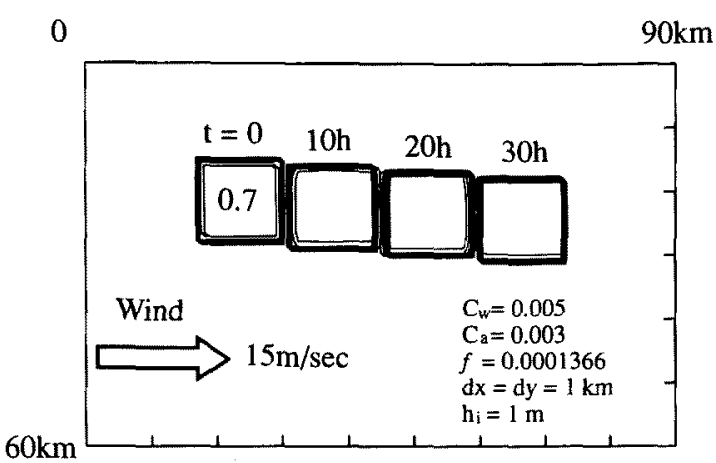

(a) Computed Ice Distribution without Ocean Flow

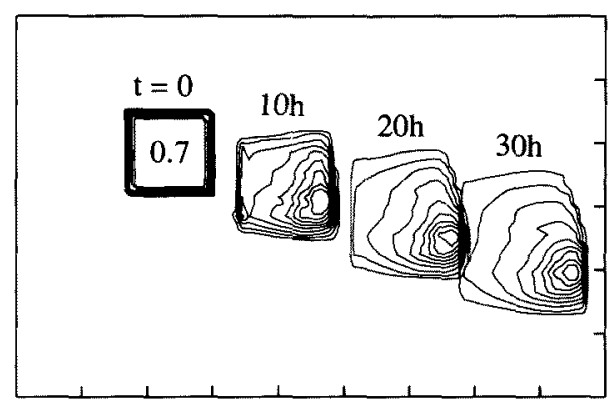

(b) Computed Ice Distribution with Ocean Flow Taken into Account

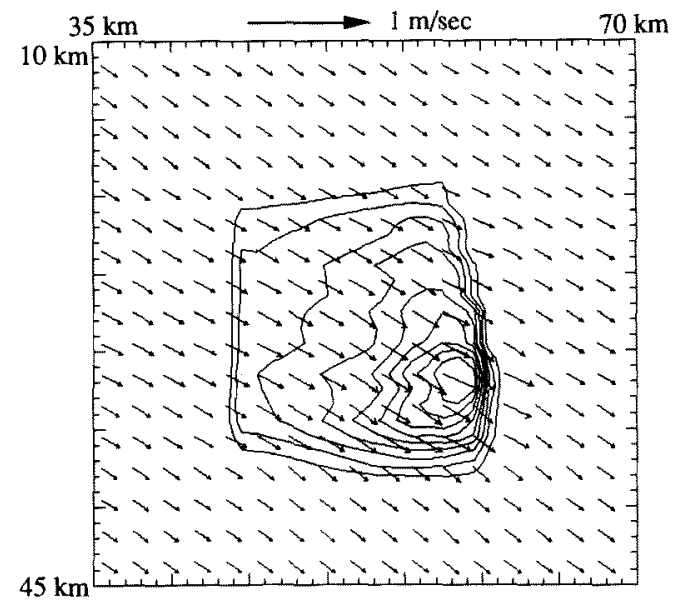

(c) Surface Water Velocity Vectors After 16 hours Together with Ice Concentration Contours

Fig. 7 Effect of Water Flow on Ice Motion

continental self partially covered with a rectangular ice group of $12 \mathrm{~km} \times 12 \mathrm{~km}$. The initial ice concentration is 0.7 , the ice/ice friction coefficient is 0.1 , and other computation parameters are given in the figure. Figure 7 (a) is for the case when the ocean current is disregrd. ed, and Figure $7(b)$ is for the case when the ocean current is taken into account. As shown in these figures, as time progresses, the ice flows downstream, slightly shifted to the right direction because of the Coriolis force. Compared with the results in Figure 7 (a) , Figure 7 (b) shows more movement of the ice. This is the effect of the water also driven by the wind. It is seen that the ice concentration increases at a downsteam right region of the ice in Figure $7(\mathrm{~b})$. Since the friction coefficients between the air and ice and also the ice and water, $C_{a}$ and $C_{w}$ are larger than that between the air and water, $C_{d}$, the water is more accelerated below the ice than out of the ice. This fact is demonstrated by Figure $7(\mathrm{c})$, where the surface water velocity vectors after 16 hours are shown with the ice concentration contours. As a result, the peak position of the ice concentration moves downstream in the ice area. Also, the diffusion of ice is due to such water velocity difference. In case that the ocean current is disregarded, the diffusion of ice and movement of the peak point of ice concentration hardly occurred as shown in Figure ? (a).

\section{Numerical Simulation in Marginal Ice Zone}

Ice floes movement is numerically simulated in mar. ginal ice zone with an ocean structure in constant water depth of $20 \mathrm{~m}$. Uniformly distributed floes on still water around a vertical rectangular structure are driven by a constant wind of $10 \mathrm{~m} / \mathrm{sec}$. The initial ice concentra. tion is 0.5 , the ice/ice friction coefficient is 0.1 , and other computation parameters are given in the Figure $8(\mathrm{a})$. The number of surface grids is $80 \times 70$ and the number of water layers is 5 . Figure 8 shows the computation results for the case when the wind blows against the face of the structure. Figure $8(\mathrm{~b})$ shows the time variation of ice concentration contours. It can be seen that the floes are accumulated in front of the structure and a part of them flows out from both sides. As a results, a region with less ice is formed behind the structure. The ice distribution becomes asymmetric beause of the Coriolis force. Figures $8(\mathrm{c})$ and $8(\mathrm{~d})$ show the ice and surface water velocity distributions after 40 minutes. It can be seen that both the flow vectors are inclined by the Coriolis force effect and the water flow carries the ice through both sides of the structure. Figure $8(\mathrm{e})$ shows the time variation of ice load on the structure. Many spike-like peaks appear due to the discrete nature of the present model : each peak corresponds to a collision of an ice bunch or bunches. Figure 9 is for the case when the wind blows at an angle of $45^{\circ}$ with the edge of the structure. The ice force on the upper side of structure in Figure $9(\mathrm{~d})$ is a little greater than that on the left side in Figure $9(\mathrm{e})$. This difference is due to the effect of Coriolis force.

\section{Conclusions}

The Distributed Mass/Discrete Floe model is a 


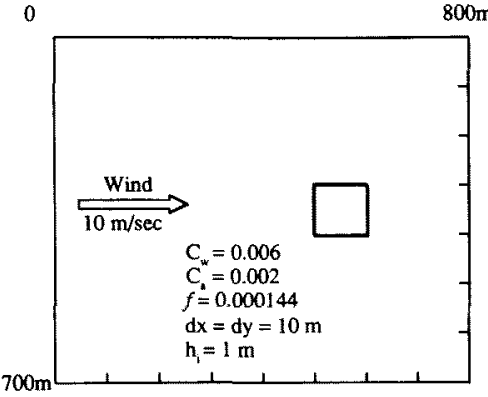

(a) Computation Dimain

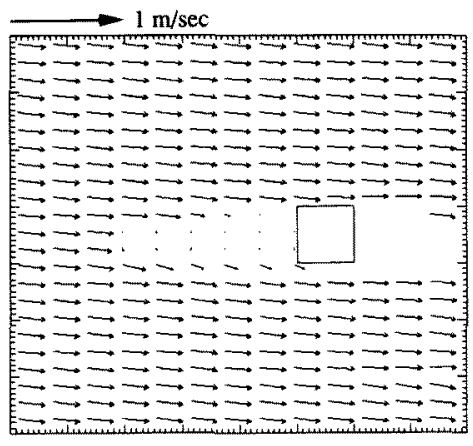

(c) Ice Velocity Distribution after 40 minutes

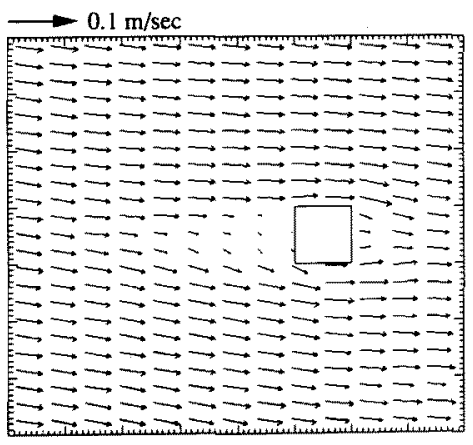

(d) Water Velocity Distribution after 40 minutes

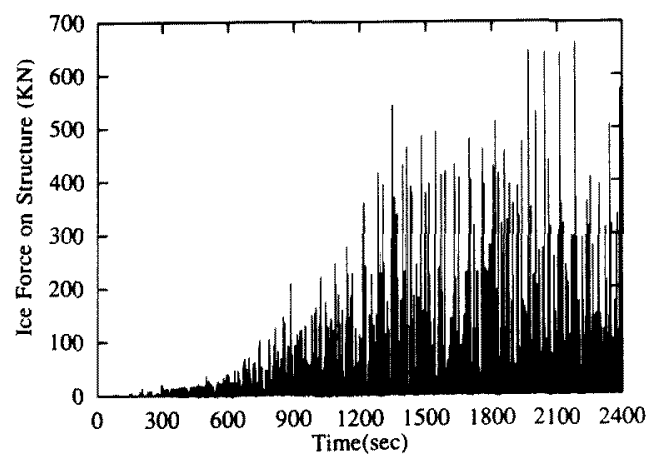

(e) Time Variation of Ice Force on the Structure
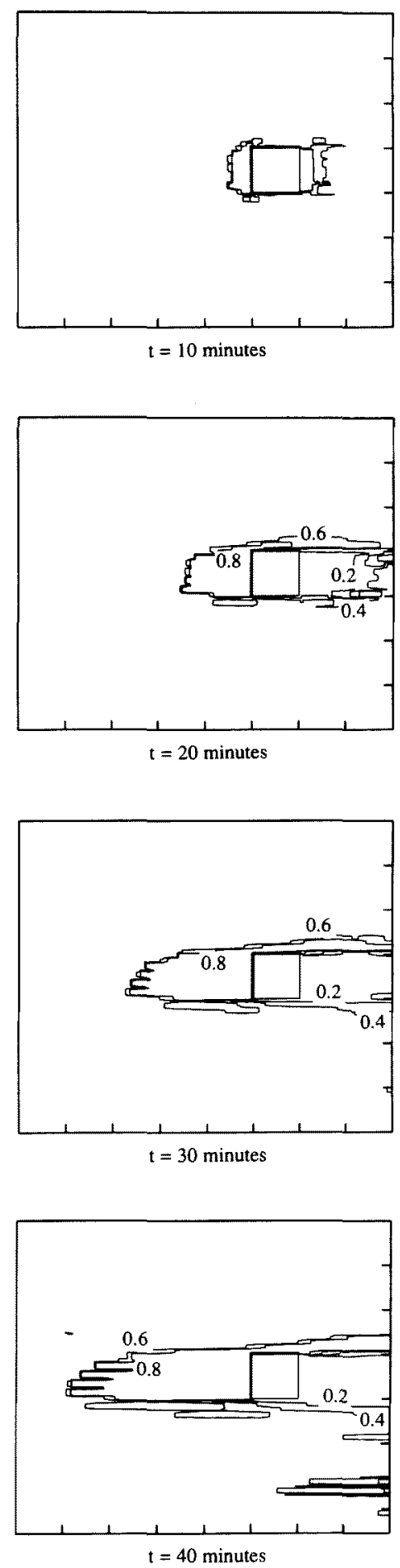

(b) Time Variation of Ice Concentration Contours

Fig. 8 Result of Wind Driven Ice/Water Combination Flow around an Rectangular Structure of $100 \mathrm{~m} \times 100 \mathrm{~m}$ Uniformly distributed ice floes are driven by a constant wind of $10 \mathrm{~m} / \mathrm{sec}$ normal to the structure face 

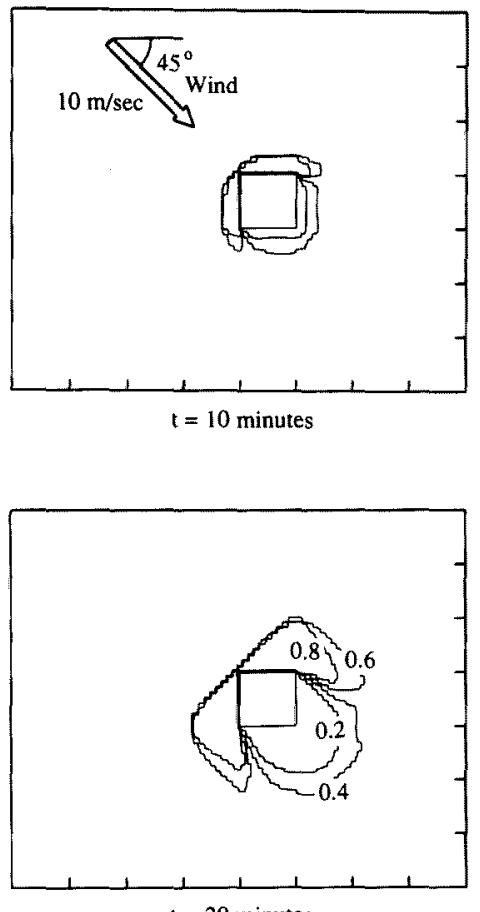

$t=20$ minutes
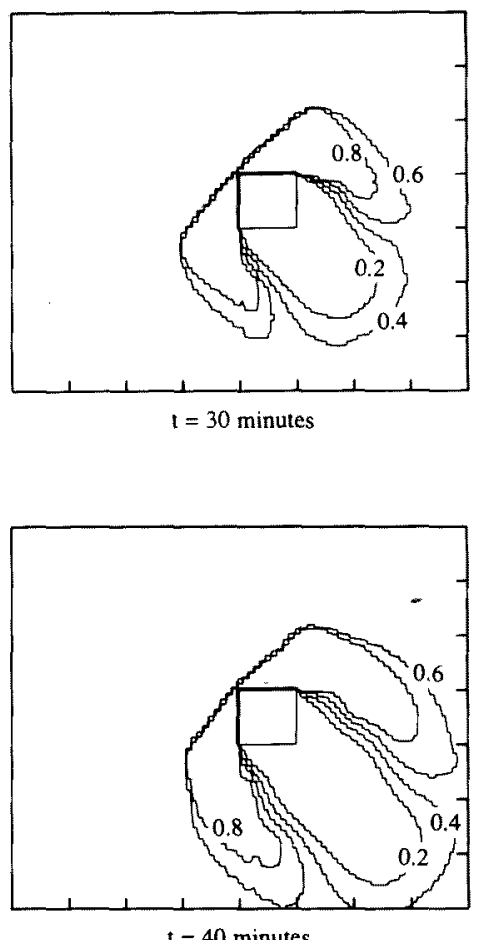

(a) Time Variation of Ice Concentration Contours

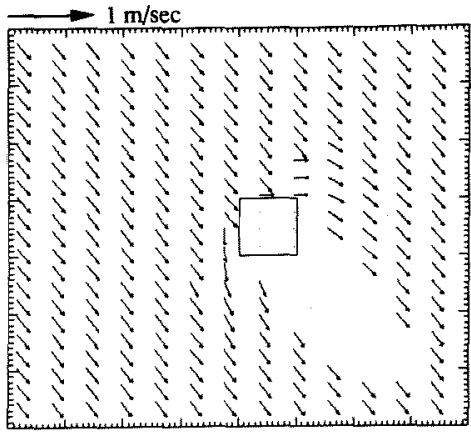

(b) Ice Velocity Distribution after 40 minutes

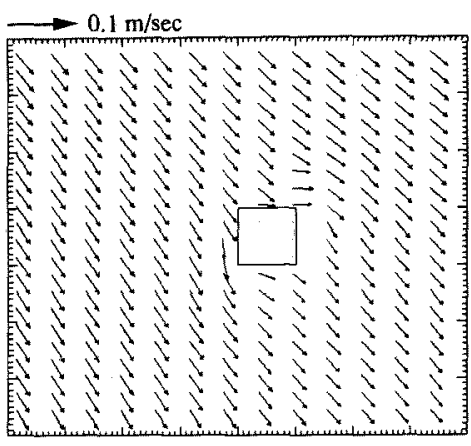

(c) Water Velocity Distribution after 40 minutes

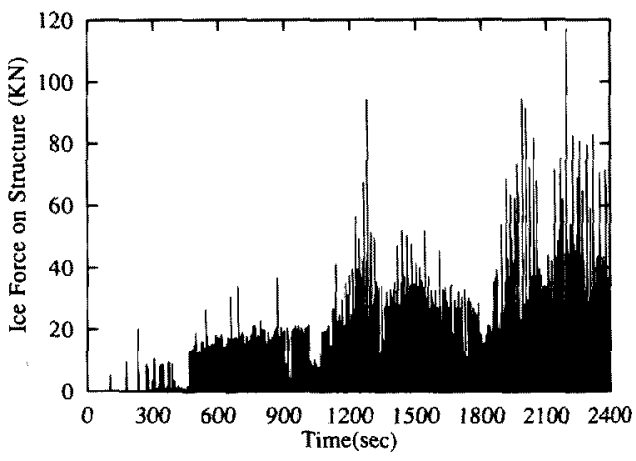

(d) Time Variation of Ice Force on the Upper Side of Structure

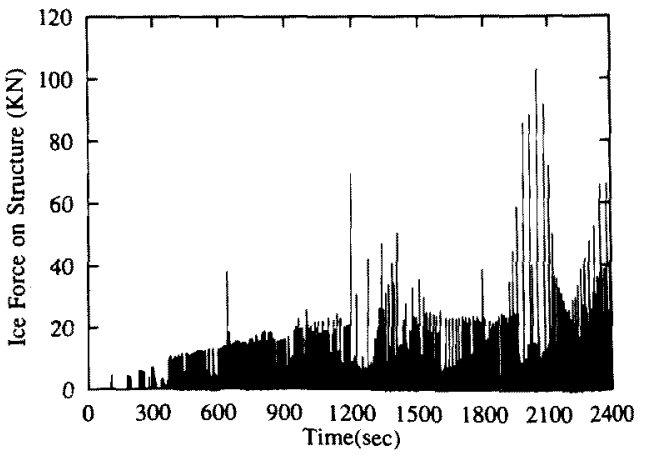

(e) Time Variation of Ice Force on the Left Side of Structure

Fig. 9 Result of Wind Driven Ice/Water Combination Flow around an Rectangular Structure of $100 \mathrm{~m} \times 100 \mathrm{~m}$; Uniformly distributed ice floes are driven by a $45^{\circ}$ inclined constant wind of $10 \mathrm{~m} / \mathrm{sec}$ 
numerical model proposed for the computation of mesoscale pack ice rheology. An improved rectangular floe version of this model was described in detail and some computations were made for simplified ocean conditions driven by a uniform wind field in the marginal ice zones. The results are summarized as follows:

1. The ice floe arrangement in a bunch and the time integral scheme for ice friction were improved. As a result, more reliable results became able to be obtained with much longer time interval of numerical integration.

2. The water surface velocity distribution is distored by the difference in the shear stress between the water/ ice interface and the water/air interface. This causes the diffusion of pack ice. As a result, the ice distribution comes to have a peak downstream of the center even if both the initial ice distribution and wind field are uniform.

3. In the case of no major current for a deep ocean, the water surface velocity can be determined from the computation only for a scale of the Ekman layer. If the water depth is less than the scale of Ekman layer, the direction of water surface flow, which is essential for the ice flow calculation, is highly affected by the water depth.

4. Many spike-like peaks appeared in the time variation of ice load on an ocean structure. This is caused by the discrete nature of the present model. Also, the results showed the rational movement of pack ice around the structure.

\section{Acknowledgments}

The authors thank the financial support of the INSROP (International Northern Sea Route Programme) organized by the Fridtjof Nansen Institute, Norway, the Central Marine Research \& Design Institute, Russia, and the Ship and Ocean Foundation, Japan.

\section{References}

1) Campbell, W. J., "The Wind-Driven Circulation of Ice and Water in a Polar Ocean", J Geophys Res, Vol. 70, No. 4, pp. 3279-3301, 1965.

2) Hibler III, W. D., "A Dynamic Thermodynamic
Sea Ice Model", J Phys Oceanogr, Vol, 9, pp. 815846, 1979.

3) Flato, G. M. and W. D. Hibler III, "Modeling Pack Ice as a Cavitating Fluid" J Phys Oceanog. raphy, Vol.22, pp.626-651, 1992.

4) Thomson N. R., J. K. Sykes and R. F. McKenna, "Short-Term Ice Motion Modeling with Application to the Beaufort Sea", J Geophys Res, Vol. 93 , pp. $6819-6836,1988$.

5) Bruno, M. S. and O. S. Madsen, "Coupled Circulation and Ice Floe Movement Model for Partially Ice-Covered Continental Shelves", J. Geophys Res, Vol. 94, pp. 2065-2077, 1989.

6) Rheem, C. K., H. Yamaguchi, H. Kato and H. Horikome, "A Numerical Study on Pack Ice Movement Using a Dynamic Ice Model as a Continuum", J Soc Naval Archi Jpn, Vol. 173, pp. 169-174, 1993.

7) Serrer, M., S. B. Savage and M. Sayed, "Visualization of Marginal Ice Zone Dynamics", Proc 1st Int Conf and Exhibition VIDEA 93, Southampton, UK, 1993.

8) Frederking, R. and M. Sayed, "Numercal Simulations of Mesoscale Rheology of Broken Ice Fields", Proc 12th Int Conf POAC, Vol. 2, pp. 789798, 1993.

9) Yamaguchi, H., C. K. Rheem and H. Kato, "Pack Ice Movement Simualation Using a Distributed Mass/Discrete Floe Model", Proc 12th Int Conf POAC, Vol. 2, pp. 848-856, 1993.

10) Rheem, C. K., H. Yamaguchi and H. Kato, "A Distributed Mass/Discrete Floe Model For Rheology Computation of Pack Ice Consisting of Disk floes", Pro 4th Int Conf ISOPE, 1994. (to appear)

11) Wu, J., "Wind-Stress Coefficients over Sea surface from Breeze to Hurricane", J Geophys Res, Vol. 87, pp. 9704-9706, 1982.

12) Thorade, H., "Die Geschwindigkeit von Triftomungen und die Ekmansche Theorie", Ann d Hydrogr u Mar Meteor, Bd. 42, pp. 379-391, 1914.

13) Hoffmann, K. A., "Computational Fluid Dynamics for Engineering", Engineering Educations System, Austin, pp. 220-221, 1989.

14) Sverdrop, H.U., M.W. Johnson and R. H. Fleming, "The Ocean", Prentice-Hall, Inc, Englewood Cliffs, NJ, pp. 489-503, 1942. 